\title{
Editorial
}

\section{Cystic fibrosis: gene therapy or preventive gene transfer?}

In 1989, the association between Cftr (the gene) and cystic fibrosis or CF (the disease) was first reported. Since then, more than 500 mutations in Cftr have been identified. CFTR (the Cftr product) has been well characterised and shown to be involved in cAMP-dependent transmembrane $\mathrm{Cl}^{-}$transport. Subsequently, several mouse Cftr knock-outs have been obtained and shown to lead to a deficiency in epithelial cell $\mathrm{Cl}^{-}$transport. Transfer of Cftr into either CFTR-deficient or CFTR-mutant cells both in vitro and in vivo has been shown to restore a normal CFTR-dependent $\mathrm{Cl}^{-}$transport. Moreover, a number of clinical studies have shown that gene transfer of wildtype human CFTR cDNA into the airways of CF patients leads to the transient restoration of the $\mathrm{Cl}^{-}$transport function and/or the transmembrane potential difference across the airway epithelium.

The accumulated knowledge undoubtedly proves that mutations in Cftr are the genetic cause of CF. However, this fact has been extended to the assumption that Cftr (and therefore CFTR) is interactive with CF along the course of the disease; although no evidence has been shown so far in support of such an assumption. The difference between 'being the cause of' and 'being interactive with' refers to the duration of the functional interaction between the elements in play, namely CFTR and CF. Although mutated CFTR causes CF, it does not prove that a lasting interaction exists between CFTR and CF; in other words, that the persistence and progression of the disease need to be continually caused by a reduced CFTR activity. We suggest that the mutant CFTR causes a primary lung dysfunction which in turn leads to a different pathological setting, no longer interactive with the CFTR activity and characterised by an autonomous kinetics.

Recent epidemiological evidence shows that the kinetics of progression of lung CF is directly related to the reduced activity of CFTR (at least for the $\Delta$ F508 and the A455E mutations) up to a point where further progression of the phenotype is independent of the CFTR activity (or the mutation on the $C f t r$ ). ${ }^{1-4}$ Based on our data, which indicate that the CFTR would be noninteractive throughout the course of the disease it causes, we postulate a new model describing the course of $\mathrm{CF}$. According to our model, lung CF would be composed of two separate disorders: CF disease followed by lung disease; which, although causally related, are not of the same nature and interact with different factors. CF disease is characterised by a physiopathological setting in the lung which is caused, and interactively maintained, by the
CFTR protein and the mucus $\mathrm{Cl}^{-}$concentration and fluidity. CF disease drives to a point from where the lung disease can be triggered. This subsequent lung disease is no longer interactive with CFTR activity nor with mucus $\mathrm{Cl}^{-}$concentration or fluidity and its kinetics is independent of the CFTR and common to all CF patients.

The border between the CF disease and the lung disease has been named as the point of no-return (PNR). Arrival at the PNR is presumably preceded by a number of cycles of bacterial proliferation/inflammation, after colonisation of the lung has occurred. ${ }^{1}$ The time that a CF lung can resist before reaching the PNR, in other words, the time that the $C F$ disease lasts, depends on genetic factors, like the CFTR-dependent rheological and bactericidal properties of the luminal mucus and on the health care (antibiotics, DNase, isolation, etc) provided to the patient. The more active the CFTR and/or better the health care: (1) the better the control on mucus fluidity and bactericidal activity; (2) the higher the first line of defence against bacterial colonisation/proliferation; and then (3) the longer the duration of the $C F$ disease preceding the lung disease.

In non-CF individuals, wild-type CFTR activity prevents bacterial colonisation of the lung; thus, the PNR is never reached and the lung disease is not triggered. Although mild mutations like A455E can significantly delay arrival of the PNR, mutant CFTR (including A455E) cannot avoid the lung reaching the PNR nor progressing into and through the lung disease.

Therefore, the role of the CFTR is limited to avoiding or delaying the arrival of the PNR, where the lung disease can be triggered. As the CF disease but not the lung disease is interactive with CFTR activity, any rational therapeutic attempts to manage the disease by manipulating CFTR activity should be restricted to the $C F$ disease stage. Thus, clinically effective CFTR gene transfer must be exclusively before the PNR.

The lung disease represents a terminal phase in the CF patient's lifespan. However, the PNR is not close to death. According to the epidemiological data, the common rate of death for lung disease CF patients is $r n=15 \%$ per year; putting the PNR roughly around at least 6-7 years before death. ${ }^{1}$ The 6-7-year span of the lung disease covers greater than $50 \%$ of the life span (approximately 10-12 years age) of Latin-American and a $20-30 \%$ of the life span of European/North American CF patients. The probability of a $\mathrm{CF}$ patient of dying within 6 years can be predicted by the score NIH. ${ }^{5}$ Score NIH values lower than 70 correspond to probabilities (of dying within 6 years) higher than 0.33 . This means that $33 \%$ of the $C F$ patients scoring less than 70 , are close to their respective 
PNR. According to this, in gene therapy clinical trials where patients with relatively high scores $\mathrm{NIH}(>70)$ are included, up to $33 \%$ of them are beyond their respective PNR. That is, for $1 / 3$ of the patients included, CFTR gene transfer does not have any rational support.

The concept of PNR has been derived from epidemiological evidence. ${ }^{1}$ As its biological nature has not been characterised, a precise definition of the border between $C F$ disease and lung disease based on clinical data is so far not possible. As the decision of whether a given patient has already passed over the PNR is not straightforward, then, inclusion criteria for recruitment of patients into clinical trials cannot be clearly derived. A clear-cut definition of the PNR, the point beyond which Cftr gene transfer is no longer meaningful, in biological and clinical terms is mandatory for an adequate selection of the patients entering clinical trials and for a rational therapeutic approach to the disease.

Apart from the clinical aspects, the preceding discussion opens critical questions about regulatory issues concerning current gene therapy approaches to CF. The review process for gene therapy clinical protocols by the regulatory authorities in the USA and in Europe is mainly based on the document known as: 'Points to Consider in the Design and Submission of Human Somatic-Cell Gene Therapy Protocols'. ${ }^{6}$ Approval of a clinical protocol is contingent on the answer to the points: the first one ('objectives and rationale of the proposed research') asks: 'Why is the disease selected for treatment by means of gene therapy as a good candidate for such treatment?'. In other words, it must be possible to make predictions on the potential effectiveness of the gene transfer on the basis of the accumulated knowledge of the disease and on the activity of the transferred gene. As the CFTR is noninteractive with the lung disease, Cftr is simply not the candidate gene for attempting therapeutic gene transfer on lung disease patients. Therefore, as long as regulatory issues are concerned, there is no rational frame to justify CFTR gene transfer into such a group of patients. The restriction of future phase II gene therapy clinical protocols to $C F$ disease patients and the exclusion of patients who have reached the PNR and are undergoing the lung disease, should be ensured by the regulatory authorities in order to comply with the 'points to consider...' regulation.

Current phase I gene therapy clinical trials are mainly (although not exclusively) addressed to the immunogenicity of the vector and the transferred gene product. Results from CF gene therapy phase I studies including lung disease patients, like most current studies, should be taken with much caution, if conclusions are to be extrapolated to the immunologically different $C F$ disease. The lung disease is characterised by repetitive cycles of bacterial proliferation/inflammation, with progressive lung tissue damage by immunomediators and immune cells. Lung disease patients do not provide the cleanest biological scenario for drawing conclusions about immunogenicity of gene transfer systems, and then, lung disease patients should preferably be excluded from toxicity studies as well. Performing phase I clinical studies on lung disease patients as a step towards future phase II trials on $C F$ disease patients, is like generating results from one pathology for making decisions on another different one. Very valuable information has been obtained from the CF gene therapy clinical trials performed so far, most of them phase I studies. However, according to the proposed model, these studies have not been focused on the right window.

The formal difference between a severe $\Delta$ F508-like and a mild A455E-like lung CF pathway is the time needed for reaching the PNR. Beyond the PNR both kinetics are the same. ${ }^{1,3}$ Therefore, for a young presymtomatic CF lung, a delay in the PNR would mimic the difference between developing a severe CF phenotype (like $\Delta$ F508) or developing a mild CF phenotype (like A455E). At the limit, if the PNR were sufficiently delayed, the CF lung might behave like a non-CF healthy carrier lung. Preventive CFTR gene transfer before the onset of the first clinical symptoms thus appears to be the ideal way to delay the PNR and the arrival of the lung disease.

According to the preceding discussion, development of a successful gene therapy approach to CF should radically switch from the current strategy, which is addressed to the therapeutic reversion of the already installed disease, into the early preventive intervention aimed at delaying the arrival of the PNR and to the lung disease. Preventive gene transfer should be initiated as early in life as possible. Optimally, it should be coupled to perinatal screening for the detection of genetically determined, but still clinically unaffected, CF patients, in order to protect them from progressing into the $C F$ disease and reaching the PNR.

As far as CF preventive gene transfer is concerned, a lasting expression of the CFTR will be required. Such a lasting expression might be achieved either by integration vectors delivered into airway epithelium progenitor cells or by repetitive applications of the (integrative or not) vector delivered into already committed airway epithelium cells. The first alternative is not feasible with the current technology and knowledge and the second is limited to the use of highly nonimmunogenic vectors. Immunogenic vectors, like current adenovirus vectors, will probably be highly restricted if not completely excluded from Cftr preventive gene transfer, whereas nonimmunogenic vectors, like for instance plasmid or synthetic DNA, might gain relevance in the development of preventive gene transfer for CF. In this context, the efficiency of the vector might be resigned in favour of a lower immunogenicity. It has recently been shown that, in accordance with the flux control theory, a small percentage correction of the Cftr genetic defect can be expected to restore the normal $\mathrm{Cl}^{-}$transepithelium potential difference genotype over the complete airway epithelium. Under the right equilibrium between the time a low efficiency vector keeps expressing the transferred gene and the frequency of repeat applications of the same vector, a cumulative effect might be expected to occur: eg sooner or later, the number of cells necessary to compensate for the whole airway epithelium, and able to cause effective prevention, should be reached.

Currently, there is a conspicuous decline in the hope that gene therapy will be able to provide a clinically effective alternative for CF in the near future. The availability of high performance (stable, efficient, nonimmunogenic, lasting expression, etc) gene transfer vectors is currently considered to be a limiting step in the further development of gene therapy in general. As most efficient gene transfer vectors are virus-derived vectors, at the same time highly immunogenic, huge efforts are made for the development of more efficient, less immunogenic viral 
vectors. The idea that successful gene therapy will have to wait until a super vector is engineered has gained ground and raised hopes.

At least for CF gene therapy, the situation is not that desperate as more rational approaches than those tested so far, are still to be explored. In future CF gene therapy clinical trials, greater attention should be paid to the study design itself. A rational study design should emphasize the following: (1) the goal ought to be early prevention (eg a delay in the PNR) instead of therapy of the lung disease; (2) the end-points have to be set by parameters indicative of prevention, for instance, a delay in the onset of bacterial colonisation or a decrease in the frequency of bacterial proliferation episodes; (3) the candidate patients entering the protocol have to be selected properly, eg much younger and as clinically presymptomatic as possible, with exclusion of lung disease patients.

In accordance with the preceding discussion of the proposed model, our group has developed a clinical protocol aimed at assessing the prevention of the arrival at the PNR. The protocol foresees the highly repeated administration of a CFTR expression plasmid vector to paediatric (6-18 years age), genetically determined but still presymptomatic, CF patients. The phase I clinical trial has received the approval of the local Committees on Clinical Research and Bioethics and awaits final approval by the ANMAT (National Food, Drug and Medical Devices Administration of Argentina).

\section{Acknowledgements}

The authors wish to thank Drs M Blaese and J Ramsey (USA), E Kremer (France) and B Groner (Germany) for fruitful discussions on the manuscript and the proposed model.
L Drittanti ${ }^{1,2,5}$, MV Masciovecchio ${ }^{1,2}$ $\mathrm{J} \mathrm{Gabbarini}^{1,4}$ and M Vega ${ }^{1,2,3,5}$ ${ }^{1}$ GTGH (Human Gene Therapy Working Group); ${ }^{2}$ Depto Biol, Bioqca y Farmacia, Universidad Nacional del Sur; ${ }^{3}$ MICROGEN S.A.-Biotechnology and ${ }^{4}$ Service of Paediatrics, Hospital Int Gral Dr J Penna, 8000-Bahía Blanca, Argentina;

5 Present address: Gene Therapy Program, Généthon II, 1 rue de l'Internationale, 91002-Evry (Paris), France

\section{References}

1 Masciovecchio MV et al. Cftr/cystic fibrosis interactivity is switched off after the initial phase of the disease. (Submitted for publication).

2 Kerem E et al. The relation between genotype and phenotype in cystic fibrosis analysis of the most common mutation (F508). New Engl J Med 1990; 323: 1517-1522.

3 Gan K-H et al. A cystic fibrosis mutation associated with mild lung disease. New Engl J Med 1995; 333: 95-99.

4 Latin American Cystic Fibrosis Patient Data, REGLAFQ. Hospital de Niños Ricardo Gutiérrez, Buenos Aires, Argentina, 1993.

5 Taussig LM, Kattwinkel J, Friedewald WT, di Sant'Agnese PA. A new prognostic score and clinical evaluation system for cystic fibrosis. J Pediatr 1973; 82: 380-390.

6 Poinst to Consider in the Design and Submission of Human Somatic-Cell Gene Therapy Protocols. National Institutes of Health/Food and Drug Administration, USA, 1996; 61 FR 10004. 